\title{
BMJ Open Physiotherapists' pain attitudes and beliefs towards chronic low back pain and their association with treatment selection: a cross-sectional study
}

\author{
Mansour Abdullah Alshehri (D) , ${ }^{1,2}$ Hosam Alzahrani (D) , ${ }^{3}$ Mazyad Alotaibi, ${ }^{4}$ \\ Ahmed Alhowimel, ${ }^{4}$ Omar Khoja ${ }^{5}$
}

To cite: Alshehri MA, Alzahrani $\mathrm{H}$, Alotaibi M, et al. Physiotherapists' pain attitudes and beliefs towards chronic low back pain and their association with treatment selection: a cross-sectional study. BMJ Open 2020;10:e037159. doi:10.1136/ bmjopen-2020-037159

- Prepublication history and additional material for this paper are available online. To view these files, please visit the journal online (http://dx.doi. org/10.1136/bmjopen-2020037159).

Received 21 January 2020 Revised 21 May 2020 Accepted 22 May 2020

Check for updates

(c) Author(s) (or their employer(s)) 2020. Re-use permitted under CC BY-NC. No commercial re-use. See rights and permissions. Published by BMJ.

For numbered affiliations see end of article.

Correspondence to Dr Mansour Abdullah Alshehri; mamshehr@uqu.edu.sa

\section{ABSTRACT}

Objectives The main aim of this study was to investigate physiotherapists' pain attitudes and beliefs towardss non-specific chronic low back pain (NSCLBP) and identify whether they are associated with treatment selection.

Design Cross-sectional study.

Setting Saudi Arabia (SA).

Participants An online survey was distributed to physiotherapists from April 2018 to January 2019. Primary and secondary outcome measures (1) Biomedical and biopsychosocial treatment orientations were assessed using the Pain Attitudes and Beliefs Scale for Physiotherapists. (2) Frequent potential treatments used by physiotherapists for individuals with NSCLBP were identified through a 20-item survey. (3) The association between physiotherapists' pain attitudes and beliefs and treatment selection was investigated. Descriptive analysis, Pearson's correlation and multinomial logistic regression were used to analyse the data using SPSS (V.26).

Results A total of 304 responses were included in the analysis. The biomedical $(34.45 \pm 7.84)$ and biopsychosocial $(31.74 \pm 5.67)$ treatment orientations were relatively low. The most frequent treatments used by physiotherapists were home exercises (87.1\%), patient education $(82.0 \%)$, specific back exercises (80.6\%), electrotherapy (61.9\%), soft tissue release $(58.8 \%)$ and spinal mobilisation or manipulation (57.8\%). Physiotherapists with a stronger biomedical treatment orientation were more likely to use treatments $(p<0.05)$ such as specific back exercises, electrotherapy, soft tissue release, hydrotherapy, massage, lumbar supports and acupuncture. However, physiotherapists with a stronger biopsychosocial treatment orientation were more likely to use cognitive functional therapy $(p<0.01)$.

Conclusions Biomedical and biopsychosocial treatment orientations were relatively low among physiotherapists in SA. Although treatments such as home exercises and patient education were frequently used, some passive and traditional treatments not recommended by clinical practice guidelines continue to be commonly used by physiotherapists in SA. This study has confirmed that physiotherapists' pain attitudes and beliefs are significantly associated with treatment selection when managing individuals with NSCLBP.

Trial registration number Researchregistry3944.
Strengths and limitations of this study

- This is the first study to investigate physiotherapists pain attitudes and beliefs towards chronic low back pain and their association with treatment selection in Saudi Arabia.

- A sample size calculation was performed and the required number of participants was achieved.

- It is an investigation into the association between physiotherapists' pain attitudes and beliefs and the selection of various potential treatments used by physiotherapists to manage individuals with chronic low back pain.

- There is potential sampling bias due to the use of convenience sampling, which might not be representative of the entire physiotherapists working in Saudi Arabia.

- Accuracy of the data is limited due to the use of a self-report survey.

\section{INTRODUCTION}

Low back pain (LBP) is one of the most widespread conditions affecting the population indiscriminately across the world. ${ }^{1}$ It is a considerable health problem and among the main causes of disability and pain. Moreover, it is a complex, multifactorial disorder and one of the most controversial conditions confronting clinicians, patients and policy-makers. Approximately 23\% of the population suffer from non-specific chronic low back pain (NSCLBP). ${ }^{2}$ Although a small proportion of people develop NSCLBP, it accounts for the majority of the LBP-related disability and economic burden. ${ }^{3}{ }^{4}$ The risk of developing NSCLBP has been associated with various physical, psychological and social factors, resulting in high levels of disability and imposing high costs on individuals and communities. $^{1 \text { 5-12 }}$

Given that physiotherapists play a key role in the management of NSCLBP, exploring their attitudes and beliefs towards NSCLBP 
and the factors that might influence them is essential. Knowing about these factors will contribute to the improvement of therapeutic strategies and, thereafter, a better treatment outcome. The existing literature has shown that factors such as the beliefs and attitudes of physiotherapists towards NSCLBP have an influence on clinical practice. ${ }^{1314}$ In addition, physiotherapists' clinical reasoning and treatment decision making are influenced by personal and working environment constraints. ${ }^{15}$ Ostelo $e t a l^{16}$ suggested two possible important sources that may influence physiotherapists' pain attitudes and beliefs towards NSCLBP: a biomedical and a biopsychosocial model. The biomedical model relies on the concept that pain and disability are consequences of physical pathology, whereas the biopsychosocial model additionally emphasises the role of psychological and social factors in the development of pain. A large percentage of physiotherapists adopt a biomedical approach (rather than a biopsychosocial approach) in their clinical diagnosis and subsequent treatment of NSCLBP. ${ }^{13}{ }^{14}$ However, such an approach might result in poorer treatment outcomes, as clinical practice guidelines recommend addressing both biopsychosocial and biomedical models in the management of people with NSCLBP. ${ }^{17} 18$ A recently published review found that therapists with a predominantly biomedical treatment orientation towards NSCLBP are more likely to advise patients to restrict their return to work duties and decrease their activity. ${ }^{13}$ However, the studies included in that review did not capture all of the treatments that are prescribed in the clinical setting and were limited only to advise on work, exercise and exercise prescription in the workplace. Therefore, there is a need for more research to investigate whether physiotherapists' beliefs and attitudes correlate with selecting treatments that are commonly prescribed for patients with NSCLBP in a clinical setting.

Another important aspect is that the pain attitudes and beliefs of physiotherapists towards NSCLBP in Saudi Arabia (SA) have not been investigated previously. This is critical for physiotherapy practice since LBP is the most common condition of musculoskeletal pain in SA among construction workers, schoolteachers and healthcare professionals. ${ }^{19-23}$ Compared with other countries, SA has a different cultural context, which has an effect on the education system, structure and curriculum of physiotherapy programmes and healthcare policies. Furthermore, with the increased use of traditional treatments for treating LBP, such as cupping therapy, ${ }^{24}$ and the limited implementation of evidence-based physiotherapy among physiotherapists, ${ }^{25}$ physiotherapy practice in SA remains a growing process. This is further complicated since many individuals with LBP in SA have limited knowledge of their condition and the related complications. ${ }^{26}$

This study aimed to: (1) explore physiotherapists' pain attitudes and beliefs towards NSCLBP, (2) identify the frequent treatments used by physiotherapists for individuals with NSCLBP and (3) investigate the association between physiotherapists' pain attitudes and beliefs and treatment selection for individuals with NSCLBP.

\section{METHODS}

\section{Design and sample}

This study used a cross-sectional design with convenience sampling.

\section{Eligibility criteria}

All participants of the study were either physiotherapists working in clinical (eg, hospitals, clinics and rehabilitation centres) or academic (eg, universities and colleges) settings in SA or physiotherapists who had just graduated and were not employed. Internship students were also invited to participate. All nationalities were considered in this study. Undergraduate students (except for internship students) were excluded.

\section{Patient and public involvement}

No patient involved.

\section{Data collection}

The study data were collected using an online survey (see the online supplementary data 1) through the SurveyMonkey website (www.surveymonkey.com). All participants' data were kept anonymous and access to the study data was limited to the authors. The survey was sent out to physiotherapists and available for participation over a 9-month timeframe (from April 2018 to January 2019). What is more, the survey was distributed through social media platforms (eg, Twitter and Facebook) and WhatsApp. To promote participation in the study, multiple reminders were sent out bi-monthly.

\section{Outcome measures}

The survey consisted of three main sections: (1) demographic information, (2) the Pain Attitudes and Beliefs Scale for Physiotherapists (PABS-PT) and (3) treatments used for individuals with NSCLBP. The first section included information (eight items) on sex, age, nationality, the highest level of education, the main work setting (eg, clinical or academic), the type of work (eg, full-time or part-time), years of experience and whether participants had received any special training in LBP.

The second section was used to examine participants' pain attitudes and beliefs towards NSCLBP. One of the most common measures used in investigating physiotherapists' pain attitudes and beliefs towards LBP is the PABSPT. The short form of the PABS-PT consists of 19 items (table 1 ) on a 6 -point scale (totally disagree $=1$ to totally agree $=6$ ) to assess two factors (maximum total score 114): 'biomedical' treatment orientation (10 items; maximum total score 60) and 'biopsychosocial' treatment orientation (9 items; maximum total score 54). ${ }^{27}{ }^{28}$ Higher scores indicate a higher level of orientation. ${ }^{28}$ The PABS-PT has reasonable validity (face and content validity) and satisfactory reliability when evaluated using Cronbach's alpha (internal consistency). ${ }^{27}$ Although alternative scales 
Table 1 A 19-item version of PABS-PT

\begin{tabular}{ll}
\hline Orientation & Items \\
\hline Biomedical & $\begin{array}{l}\text { 1. The severity of tissue damage } \\
\text { determines the level of pain. }\end{array}$
\end{tabular}

2. Increased pain indicates new tissue damage or the spread of existing damage.

3. Pain is a nociceptive stimulus, indicating tissue damage.

4. If back pain increases in severity, I immediately adjust the intensity of my treatment accordingly.

5. If patients reported of pain during exercise, I worry that damage is being caused.

6. Patients with back pain should preferably practice only pain-free movements.

7. Pain reduction is a precondition for the restoration of normal functioning.

8. If therapy does not result in a reduction in back pain, there is a high risk of severe restrictions in the long term.

9. Back pain indicates the presence of organic injury.

10. In the long run, patients with back pain have a higher risk of developing spinal impairments.

Biopsychosocial 11. Learning to cope with stress promotes recovery from back pain.

12. A patient suffering from severe back pain will benefit from physical exercise.

13. Even if the pain has worsened, the intensity of the next treatment can be increased.

14. Exercises that may be back straining should not be avoided during the treatment.

15. Therapy may have been successful even if the pain remains.

16. The cause of back pain is unknown.

17. Functional limitations associated with back pain are the result of psychosocial factors.

18. There is no effective treatment to eliminate back pain.

19. Mental stress can cause back pain even in the absence of tissue damage.

PABS-PT, Pain Attitudes and Beliefs Scale for Physiotherapists.

assessing pain attitudes are available, the PABS-PT has two advantages over other scales, including that (a) these items were developed specifically for physiotherapists and, therefore, could be more representative of this group and (b) this scale considers the outcomes of two dimensions (biomedical and psychosocial factors) in comparison with other scales with only one outcome dimension since physiotherapists may have a treatment orientation that is not suitable for placement on one dimension. Considering biomedical and psychosocial issues together is important because of the nature of NSCLBP. Thus, the PABS-PT provides more information in terms of therapists' treatment orientation.

The final section included a question on physiotherapy practice in SA in terms of frequent treatments used for individuals with NSCLBP, covering all potential treatments (20 items). All 20 items were carefully selected based on the physiotherapy practice as reported in the literature. The survey was written in the English language, as it is considered the formal language used in healthcare education and practice in SA. Furthermore, the survey was revised multiple times by the authors in order to assess the relevance and clarity of the included questions and correct mistakes and typos in the text.

\section{Sample size calculation}

According to a recent statistical report conducted by the Saudi Commission for Health Specialties (SCFHS) in 2018, the total number of registered/licensed physiotherapists in SA was 6028. This estimated number includes Saudi $(n=4410)$ and non-Saudi $(n=1618)$ physiotherapists who are working in governmental and private health sectors. ${ }^{29}$ Our study considered those who had newly graduated, which was estimated by the SCFHS to be 940 students in 2018. Moreover, our study considered those who had not renewed their registration/licence. Consequently, considering all of these factors, we proposed that the total number of physiotherapists in SA is between 7500 and 8000 . The sample size was calculated by setting the statistical power at a $90 \% \mathrm{CI}$, with a population size of 8000 and a margin of error of $5 \%$. Thus, the required sample size for this study constituted 261 participants.

\section{Statistical data analysis}

Incomplete responses in which only demographic information was available were excluded. Descriptive statistical analysis (including frequency and percentage) was used to observe the distribution of participants' responses. In addition, the mean and SD were used to calculate the scores of the PABS-PT. What is more, Pearson's correlation coefficient was used to examine the correlation between the biomedical and biopsychosocial subscales of the PABS-PT. An OR with a 95\% CI using multinomial logistic regression analysis was used to investigate (1) the association between PABS-PT scores and participants' characteristics, (2) the association between participants' characteristics and treatment selection, and (3) the association between PABS-PT scores and treatment selection. All calculated ORs were adjusted for all potential confounding factors/variables (sex, age, nationality, education, main work setting, type of main work, years of experience and special training in LBP). The findings 


\begin{tabular}{|c|c|c|}
\hline Variables & & N (\%) \\
\hline \multirow[t]{2}{*}{ Sex } & Male & $188(61.8)$ \\
\hline & Female & $116(38.2)$ \\
\hline \multirow[t]{5}{*}{ Age (years) } & $18-25$ & $84(27.6)$ \\
\hline & $26-30$ & $90(29.6)$ \\
\hline & $31-35$ & $55(18.1)$ \\
\hline & $36-40$ & $41(13.5)$ \\
\hline & $\geq 41$ & $34(11.2)$ \\
\hline \multirow[t]{2}{*}{ Nationality } & Saudi & $252(82.9)$ \\
\hline & Non-Saudi & $52(17.1)$ \\
\hline \multirow[t]{2}{*}{ Education } & $\begin{array}{l}\text { Undergraduate } \\
\text { (Diploma, BSc or DPT) }\end{array}$ & $187(61.5)$ \\
\hline & Postgraduate (MSc or PhD) & $117(38.5)$ \\
\hline \multirow[t]{3}{*}{ Main work setting } & Clinical setting & $195(64.1)$ \\
\hline & Academic setting & $75(24.7)$ \\
\hline & $\begin{array}{l}\text { Newly graduated/ } \\
\text { unemployed }\end{array}$ & $34(11.2)$ \\
\hline \multirow[t]{3}{*}{ Type of main work } & Full-time & $244(80.3)$ \\
\hline & Part-time & $24(7.9)$ \\
\hline & $\begin{array}{l}\text { Newly graduated/ } \\
\text { unemployed }\end{array}$ & $36(11.8)$ \\
\hline \multirow[t]{2}{*}{ Years of experience } & $1-10$ & $219(72.0)$ \\
\hline & $\geq 11$ & $85(28.0)$ \\
\hline \multirow{2}{*}{$\begin{array}{l}\text { Special training in } \\
\text { LBP }\end{array}$} & Yes & $218(71.7)$ \\
\hline & No & $86(28.3)$ \\
\hline
\end{tabular}

BSc, Bachelor of Science; DPT, Doctor of Physical Therapy; LBP, low back pain; MSc, Master of Science; N (\%), number of participants (percentage); PhD, Doctor of Philosophy.

were considered statistically significant when the $p$ value was $<0.05$. The data were analysed using IBM SPSS V.26 (IBM Corp).

\section{RESULTS \\ Demographic information}

A total of 304 responses were received in the study and had complete information for the first two sections of the survey, of which 294 had complete information for all of the survey sections. The majority of the participating physiotherapists were male individuals $(61.8 \%)$, below 30 years of age $(57.2 \%)$ and their nationality was Saudi $(82.9 \%)$. More than half of the physiotherapists $(54.3 \%)$ had completed their bachelor's degree and more than one-quarter of them (28.6\%) had a master's degree. The majority of the physiotherapists worked in clinical settings $(64.1 \%)$ and more than two-thirds $(80.3 \%)$ worked full-time. Approximately two-thirds of the physiotherapists $(72.0 \%)$ had less than 10 years of experience. Many of the participating physiotherapists $(71.7 \%)$ had received special training in LBP management. Table 2 demonstrates the demographic information of the participants.

\section{Physiotherapists' pain attitudes and beliefs towards NSCLBP}

The overall mean score for biomedical treatment orientation was 34.45 (SD 7.84) and for biopsychosocial treatment orientation was 31.74 (SD 5.67). The overall mean score for both treatment orientations was 66.19 (SD 10.04). Among the 19 items of the PABS-PT, the three lowest mean score items (13, 16 and 18) were related to biopsychosocial treatment orientation (figure 1). Regarding physiotherapists who 'largely' or 'totally' agreed with a statement (item), item numbers 4 and 7 for biomedical treatment orientation and item numbers 12 and 19 for biopsychosocial treatment orientation were the highest in terms of physiotherapists' beliefs (table 3). In biomedical treatment orientation, $40.8 \%$ of physiotherapists believed that the intensity of treatment should be adjusted if the severity of pain is increased, whereas $48.1 \%$ of them believed that the reduction of pain is a prerequisite for restoring normal functioning. In terms of biopsychosocial treatment orientation, more than half of the physiotherapists $(59.2 \%)$ believed (largely or totally agreed) that physical exercise can be beneficial for individuals with severe LBP. Moreover, the majority of them $(60.5 \%)$ believed that LBP can be caused by mental stress, even with no tissue damage.

There were significant associations between physiotherapists' PABS-PT scores in relation to some variables of demographic information (table 4). ORs showed that stronger biomedical treatment orientation was less likely in Saudi physiotherapists than in non-Saudi physiotherapists $(\mathrm{p}<0.01)$. Interestingly, stronger biomedical treatment orientation was less likely where stronger biopsychosocial treatment orientation was more likely in those who had received special training in LBP $(p<0.05)$. This study did not find a correlation between biomedical and biopsychosocial subscales of the PABS-PT $\left(\mathrm{R}^{2} 0.007\right)$.

\section{Frequent treatments used by physiotherapists for NSCLBP}

The most frequent treatments (figure 2) used by physiotherapists for NSCLBP were home exercises $(87.1 \%)$, patient education (82.0\%), specific back exercises $(80.6 \%)$, electrotherapy $(61.9 \%)$, soft tissue release $(58.8 \%)$ and spinal mobilisation or manipulation $(57.8 \%)$. The less frequent treatments used were the 'pathoanatomic-based classification' approach (5.4\%), the 'movement system impairment (MSI)-based classification' approach (11.2\%), cupping therapy (13.3\%), acupuncture $(13.9 \%)$ and the 'treatment-based classification' approach $(18.0 \%)$.

Some demographic variables were observed to have a significant association with treatment selection (table 5). For example, male physiotherapists were more likely to advise bed rest (OR 1.92; 95\% CI: 1.01 to 3.63 ; $\mathrm{p}<0.05$ ) than were female physiotherapists. Meanwhile, they (male physiotherapists) were less likely to use treatments such as patient education (OR 0.48 ; $95 \%$ CI: 0.23 to 0.99 ; 


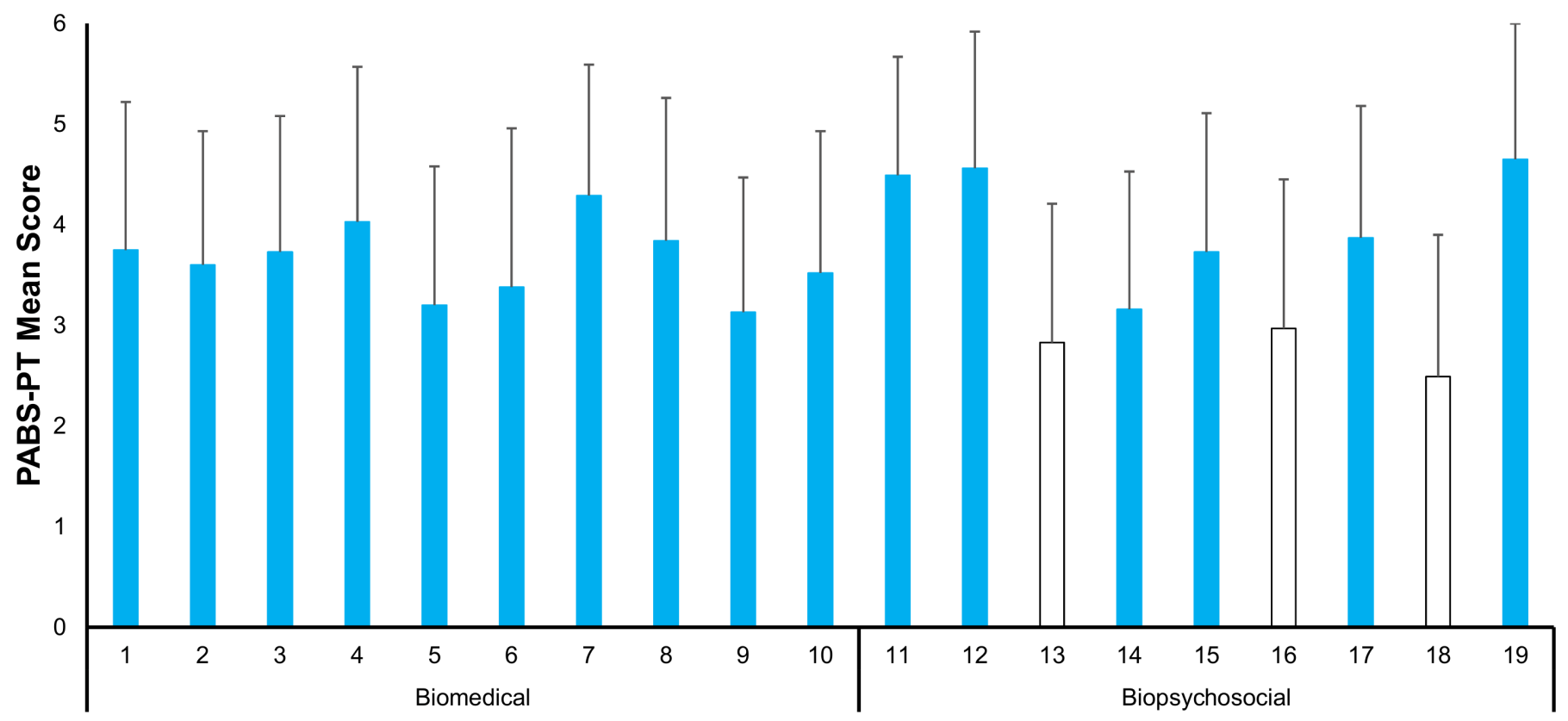

PABS-PT Items

Figure 1 Mean scores of all PABS-PT items (19 items). The three lowest mean score items were related to biopsychosocial treatment orientation, which are shown as white bars. The vertical line refers to the SD. PABS-PT, Pain Attitudes and Beliefs Scale for Physiotherapists.

\begin{tabular}{|c|c|c|c|c|c|c|c|}
\hline \multirow[b]{2}{*}{ Orientation } & \multirow[b]{2}{*}{ Items } & \multicolumn{6}{|l|}{ N (\%) } \\
\hline & & $\begin{array}{l}\text { Totally } \\
\text { disagree }\end{array}$ & $\begin{array}{l}\text { Largely } \\
\text { disagree }\end{array}$ & $\begin{array}{l}\text { Disagree to } \\
\text { some extent }\end{array}$ & $\begin{array}{l}\text { Agree to } \\
\text { some extent }\end{array}$ & $\begin{array}{l}\text { Largely } \\
\text { agree }\end{array}$ & Totally agree \\
\hline \multirow[t]{7}{*}{ Biomedical } & 1 & 33 (10.9) & 35 (11.5) & 35 (11.5) & $111(36.5)$ & 53 (17.4) & 37 (12.2) \\
\hline & 2 & $28(9.2)$ & $38(12.5)$ & $52(17.1)$ & $118(38.8)$ & $47(15.5)$ & $21(6.9)$ \\
\hline & 4 & $30(9.9)$ & $25(8.2)$ & $36(11.8)$ & $89(29.3)$ & $62(20.4)$ & $62(20.4)$ \\
\hline & 5 & 40 (13.2) & $54(17.8)$ & $84(27.6)$ & $76(25.0)$ & $30(9.9)$ & $20(6.6)$ \\
\hline & 6 & $54(17.8)$ & $40(13.2)$ & $58(19.1)$ & $71(23.4)$ & $51(16.8)$ & $30(9.9)$ \\
\hline & 7 & $16(5.3)$ & $17(5.6)$ & $28(9.2)$ & 97 (31.9) & 95 (31.3) & $51(16.8)$ \\
\hline & 8 & $25(8.2)$ & $32(10.5)$ & $52(17.1)$ & $89(29.3)$ & $71(23.4)$ & 35 (11.5) \\
\hline \multirow{8}{*}{ Biopsychosocial } & 12 & $17(5.6)$ & $10(3.3)$ & $24(7.9)$ & $73(24.0)$ & $94(30.9)$ & $86(28.3)$ \\
\hline & 13 & $65(21.4)$ & $64(21.1)$ & $81(26.6)$ & $62(20.4)$ & $17(5.6)$ & $15(4.9)$ \\
\hline & 14 & 44 (14.5) & $55(18.1)$ & $75(24.7)$ & $84(27.6)$ & $30(9.9)$ & $16(5.3)$ \\
\hline & 15 & $29(9.5)$ & $30(9.9)$ & $53(17.4)$ & $100(32.9)$ & $67(22.0)$ & $25(8.2)$ \\
\hline & 16 & $64(21.1)$ & 65 (21.4) & $53(17.4)$ & 77 (25.3) & $28(9.2)$ & $17(5.6)$ \\
\hline & 17 & $21(6.9)$ & $27(8.9)$ & $44(14.5)$ & $124(40.8)$ & $56(18.4)$ & $32(10.5)$ \\
\hline & 18 & 97 (31.9) & $79(26.0)$ & $51(16.8)$ & $47(15.5)$ & $20(6.6)$ & $10(3.3)$ \\
\hline & 19 & $14(4.6)$ & $13(4.3)$ & $18(5.9)$ & $75(24.7)$ & $84(27.6)$ & $100(32.9)$ \\
\hline
\end{tabular}

N (\%), number of participants (percentage); NSCLBP, non-specific chronic low back pain; PABS-PT, Pain Attitudes and Beliefs Scale for Physiotherapists. 
Table 4 Association between PABS-PT scores and physiotherapists' characteristics

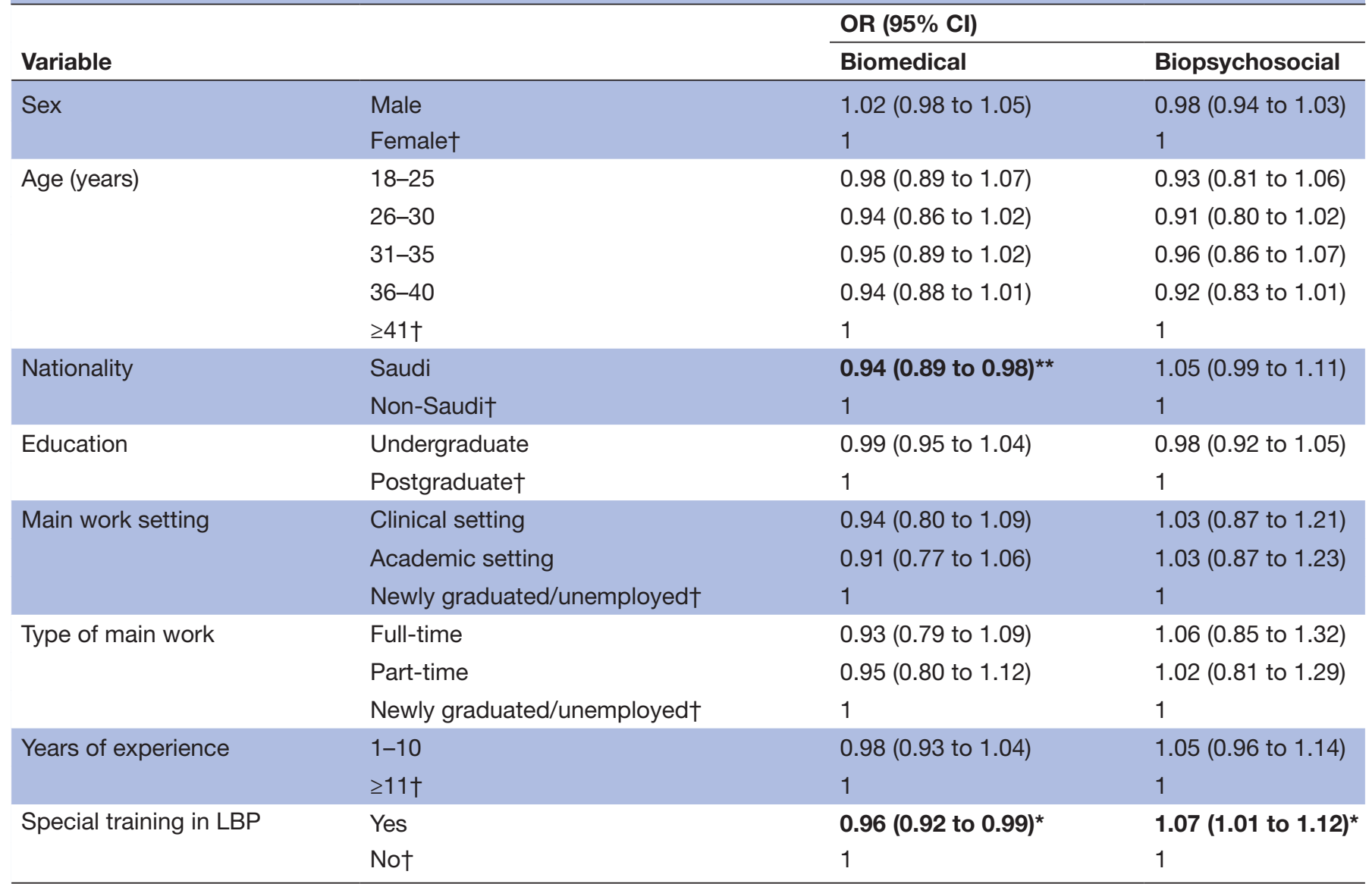

ORs with $95 \%$ Cls were used as a measure of association and were adjusted for all variables in the table (sex, age, nationality, education, main work setting, type of main work, years of experience and special training in LBP).

$P$ values of statistically significant associations are given in bold $\left({ }^{\star} p<0.05 ;{ }^{* \star} p<0.01\right)$.

†The reference category.

LBP, low back pain; PABS-PT, Pain Attitudes and Beliefs Scale for Physiotherapists.

$\mathrm{p}<0.05)$, specific back exercises (OR 0.27; $95 \%$ CI: 0.13 to $0.60 ; \mathrm{p}<0.01)$ and physical-activity-based interventions (OR 0.51 ; $95 \%$ CI: 0.30 to 0.87 ; $\mathrm{p}<0.05$ ). Junior physiotherapists (18-25 years old) were more likely to use soft tissue release (OR 6.41; 95\% CI: 1.56 to $26.40 ; \mathrm{p}<0.05)$ and spinal traction (OR $6.26 ; 95 \%$ CI: 1.35 to $29.03 ; \mathrm{p}<0.05$ ) than were senior physiotherapists ( $\geq 41$ years old). Physiotherapists who had received special training in LBP were more likely to use physical-activity-based interventions (OR 1.91; 95\% CI: 1.08 to 3.39 ; $\mathrm{p}<0.05$ ) and less likely to advise bed rest (OR 0.53 ; 95\% CI: 0.27 to 1.00 ; $\mathrm{p}=0.051$ ) than were those who had not received training in LBP. See the online supplementary data 2 for all significant and non-significant associations between demographic variables and treatment selection.

\section{The association between physiotherapists' pain attitudes and} beliefs and treatment selection

There were significant associations between physiotherapists' PABS-PT scores in relation to treatment selection (figure 3). Physiotherapists with stronger biomedical treatment orientation were more likely to use treatments such as specific back exercises $(\mathrm{p}<0.05)$, electrotherapy $(\mathrm{p}<0.05)$, soft tissue release $(\mathrm{p}<0.001)$, hydrotherapy $(\mathrm{p}=0.057)$, massage $(\mathrm{p}<0.01)$, lumbar supports $(\mathrm{p}<0.05)$ and acupuncture $(\mathrm{p}=0.051)$. In addition, those with stronger biomedical treatment orientation were less likely to use treatments such as physical-activity-based interventions $(\mathrm{p}<0.05)$, pain-coping skills training $(\mathrm{p}<0.05)$ and cognitive functional therapy $(\mathrm{p}<0.01)$. Meanwhile, physiotherapists with stronger biopsychosocial treatment orientation were more likely to use cognitive functional therapy $(\mathrm{p}<0.01)$ and less likely to use lumbar supports $(\mathrm{p}<0.05)$. See the online supplementary data 3 for the associations between physiotherapists' PABS-PT scores and treatment selection (including ORs with 95\% CI values).

\section{DISCUSSION}

This study found that biomedical and biopsychosocial treatment orientations were relatively low among physiotherapists in SA based on PABS-PT scores. The most frequent treatments used by physiotherapists for NSCLBP were home exercises, patient education, specific back 


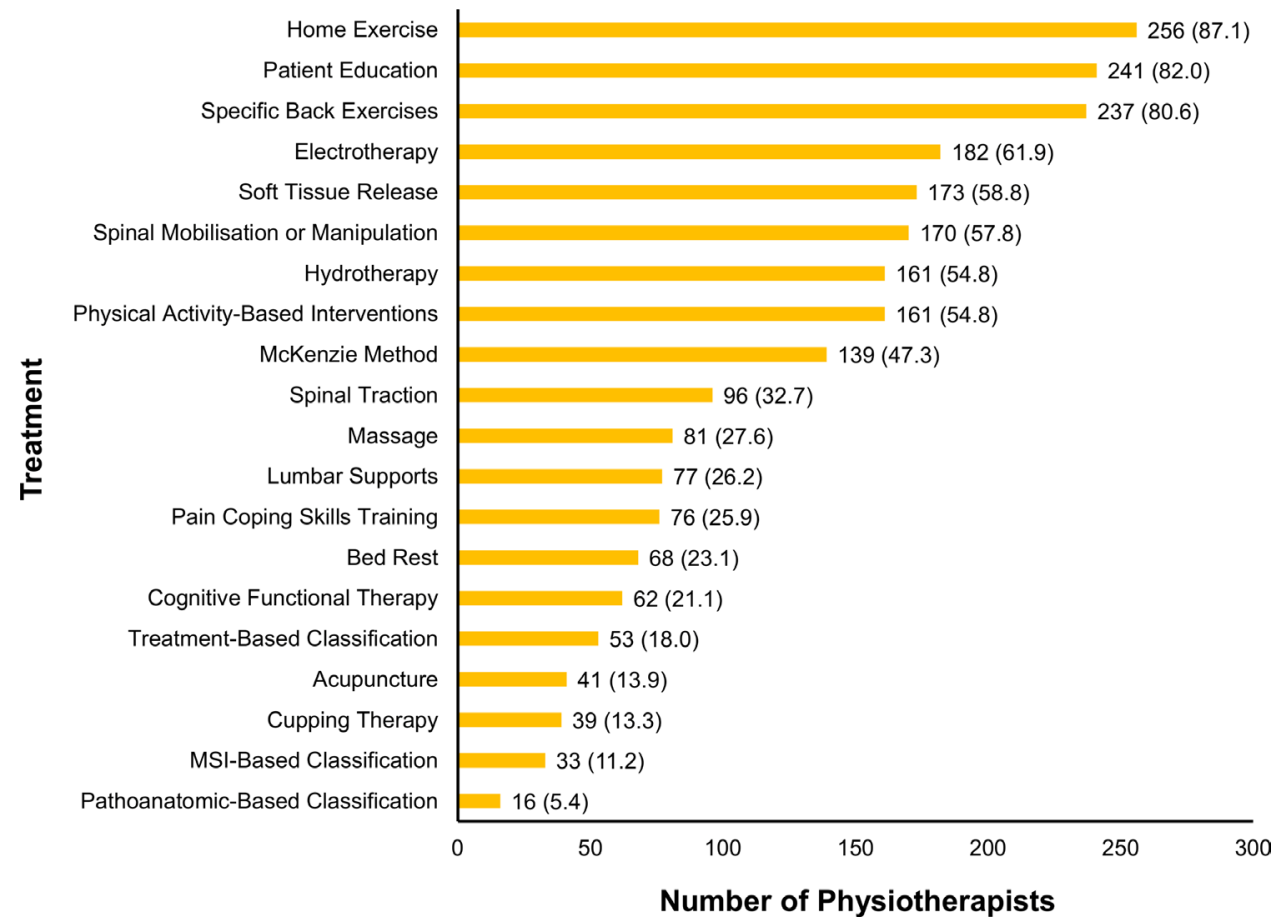

Figure 2 Frequency distribution of treatments used by physiotherapists for NSCLBP. MSI, movement system impairment; NSCLBP, non-specific chronic low back pain.

exercises, electrotherapy, soft tissue release and spinal mobilisation. There were some significant associations between physiotherapists' PABS-PT scores in relation to demographic information such as physiotherapists who had received special training in LBP. What is more, some demographic variables were observed to have a significant association with treatment selection, such as sex, age, type of work and special training in LBP. The main findings of this study confirmed that there were significant associations between physiotherapists' PABS-PT scores in relation to treatment selection. Physiotherapists with stronger biomedical treatment orientation were more likely to use treatments such as specific back exercises, electrotherapy, soft tissue release, hydrotherapy, massage, lumbar supports and acupuncture. Meanwhile, physiotherapists with stronger biopsychosocial treatment orientation were more likely to use cognitive functional therapy and less likely to use lumbar supports. This suggests that physiotherapists' pain attitudes and beliefs are critical factors which might have an impact on treatment selection for individuals with NSCLBP.

\section{Physiotherapists' pain attitudes and beliefs towards NSCLBP}

Originally, the PABS-PT scores were divided into two main dimensions: biomedical orientation and biopsychosocial orientation. The results of the current study demonstrated that the estimated physiotherapists' scores on the two subscales of the short version of the PABS-PT (19 items) were within the ranges of some of the past studies, but also different from those of other studies that used a similar version of the PABS-PT. The results of physiotherapists' PABS-PT scores (mean \pm SD) in our study were similar to those of physiotherapists working in the UK (biomedical=31.1 \pm 7.2 ; biopsychosocial $=32.5 \pm 4.8),{ }^{30} \quad$ Canada $\quad$ (biomedical $=31.14 \pm 6.67$; biopsychosocial $=32.08 \pm 4.83)^{31}$ and New Zealand (biomedical $=31.12 \pm 6.67$; biopsychosocial $=31.76 \pm 4.30){ }^{32}$ Moreover, the results were similar to those of Innes $e \mathrm{al}^{33}$ who found that the mean scores of biomedical and biopsychosocial subscales were $34.5 \pm 6.3$ and $31.4 \pm 4.1$ among Australian chiropractors, respectively. In the present study, the mean scores of biomedical and biopsychosocial subscales were $34.45 \pm 7.84$ and $31.74 \pm 5.67$ among participating physiotherapists, respectively. However, some other studies reported slightly different results. For example, Houben $e t a l^{27}$ reported an overall mean score of $29.5 \pm 7.9$ for the biomedical subscale and 35.6 \pm 5.6 for the biopsychosocial subscale among Dutch healthcare professionals, indicating that the biomedical subscale score was significantly lower than the biopsychosocial subscale score. Meanwhile, another study conducted by Magalhães et $a l^{34}$ reported that both biomedical $(27.06 \pm 7.19)$ and biopsychosocial $(24.34 \pm 6.31)$ scores were very low among Brazilian physiotherapists. However, the study conducted by Houben $e t a l^{27}$ not only was limited to physiotherapists, but also included other healthcare professionals such as chiropractors, manual therapists and osteopaths. Moreover, this study did not perform a subgroup analysis to consider the differences in pain attitudes and beliefs between the included healthcare professionals. Meanwhile, the study conducted by Magalhães $e t a l^{34}$ included a sample size that was smaller than those of previous studies and the majority of their participants were juniors with regard to experience. 
Table 5 Association between physiotherapists' characteristics and treatment selection

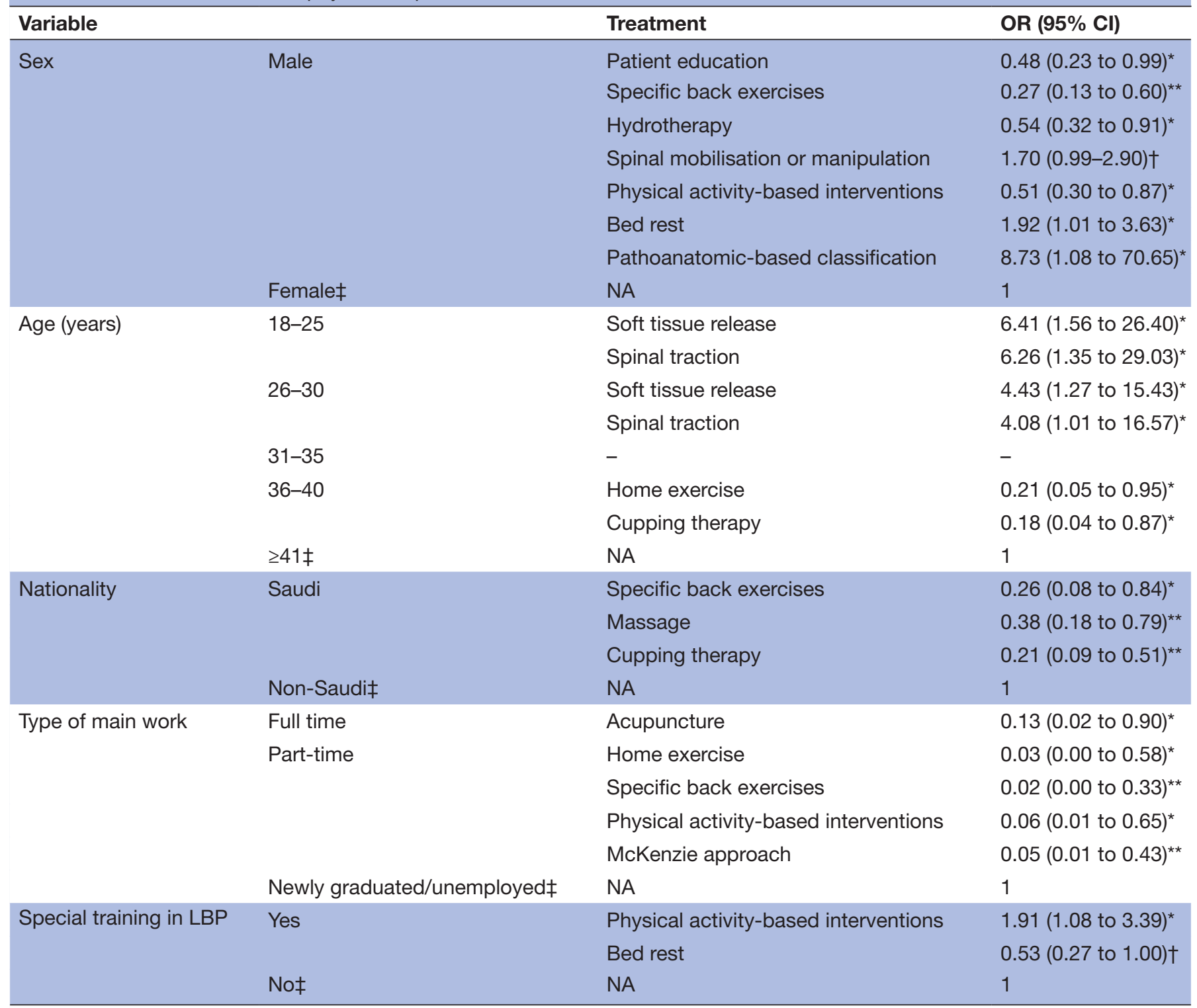

ORs with $95 \% \mathrm{Cls}$ were used as a measure of association and were adjusted for all variables in the table (sex, age, nationality, education, main work setting, type of main work, years of experience and special training in LBP).

Only significant associations $\left({ }^{*} \mathrm{p}<0.05 ;{ }^{* \star} \mathrm{p}<0.01\right.$ ) were reported in this table (see the online supplementary data 2 for all significant and nonsignificant associations).

†Amarginal trend towards significance $(p=0.050-0.053)$.

$\ddagger$ The reference category.

NA, not applicable; LBP, low back pain.

Few studies investigated whether there was an association between the demographic variables of physiotherapists and their biomedical or biopsychosocial orientations. Magalhães $e t a \vec{l}^{4}$ found a significant association between male and less experienced physiotherapists and the use of the biomedical approach in the management of individuals with NSCLBP. In our study, the majority of demographic information (eg, sex, age or years of experience) was not associated with biomedical and biopsychosocial orientations. Meanwhile, physiotherapists who had received special training in LBP were more likely to have higher scores on the biopsychosocial subscale than were counterparts who had not received special training in LBP. However, not all studies reported similar results. For example, Innes $e t a l^{33}$ did not find significant associations between demographic variables and PABS-PT subscales (biomedical and biopsychosocial).

Several studies investigated the correlation between biomedical and biopsychosocial orientations towards NSCLBP in physiotherapists. These studies found an inverse correlation between both orientations based on PABS-PT scores. ${ }^{30-33}$ This implies that physiotherapists who had higher scores on one subscale of the PABS-PT were likely to have lower scores on the other 


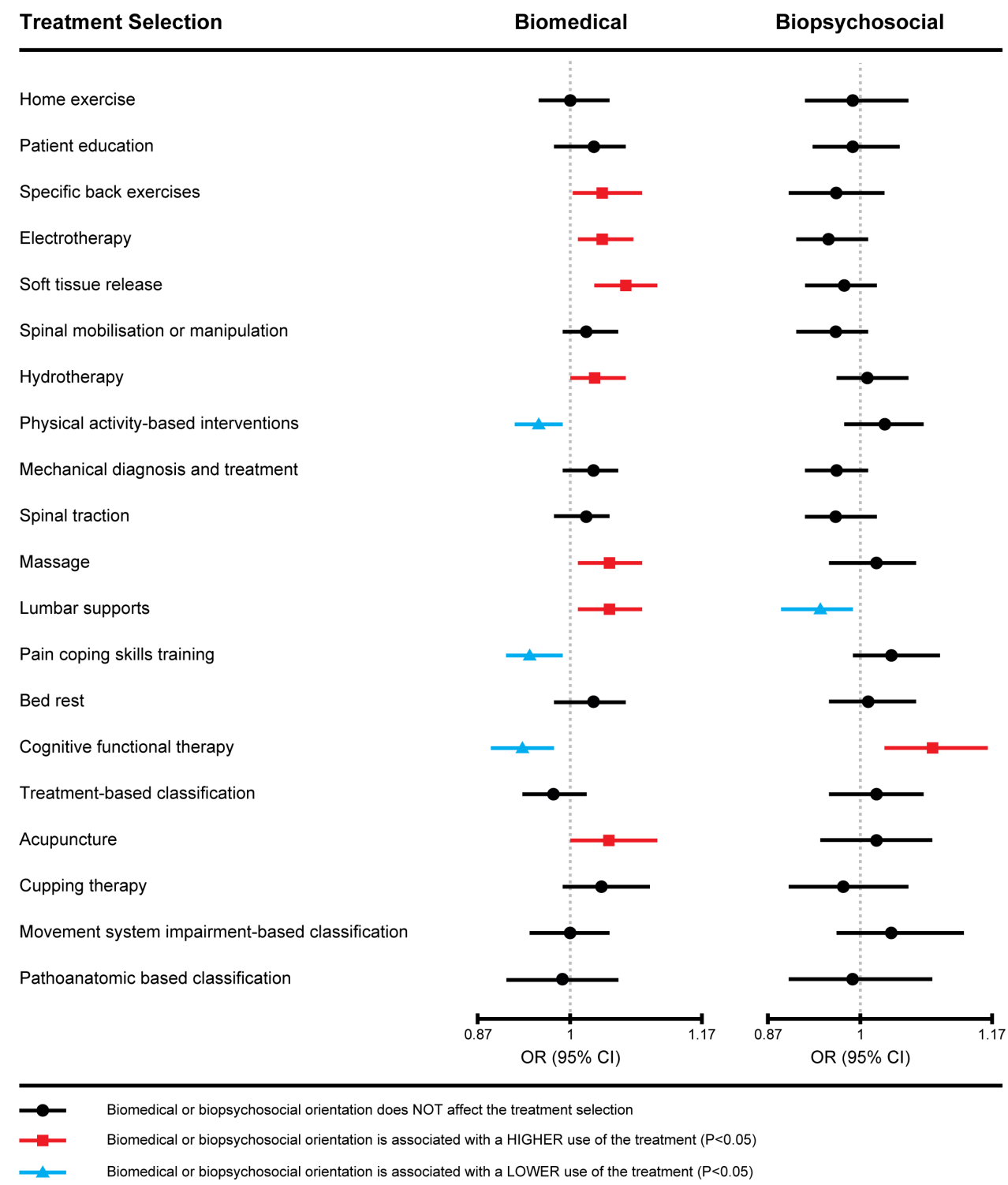

Figure 3 Association between PABS-PT scores (biomedical and biopsychosocial treatment orientation) and treatment selection. The results were presented in the form of ORs with $95 \% \mathrm{Cls}$ as a measure of association. ORs presented in the figure were adjusted for sex, age, nationality, education, main work setting, type of main work, years of experience and special training received in LBP. Statistically significant associations were printed in colour (with square and triangular plots). LBP, low back pain; PABS-PT, Pain Attitudes and Beliefs Scale for Physiotherapists.

subscale. In contrast, our study did not find a correlation between the biomedical and biopsychosocial subscales of the PABS-PT, which is inconsistent with the results of previous studies. The difference between our study and past studies regarding the scores of PABS-PT subscales could be explained by differences in demographic variables, different cultural backgrounds, different academic training and different levels of professional experience. Another possible explanation is the inclusion of unemployed or newly graduated physiotherapists in our study compared with other studies. Moreover, the differences in the structure/curriculum of physiotherapy programmes in Saudi universities compared with developed countries (eg, the USA, the UK and Australia) may have been a factor: in the former, the biopsychosocial model is rarely considered and less frequently taught in undergraduate physiotherapy programmes, whereas in the latter, the biopsychosocial model has been well acknowledged and often implemented.

\section{Frequent treatments used by physiotherapists for NSCLBP}

Few studies have investigated the treatment strategies used by physiotherapists in the management of LBP. Furthermore, the results of the majority of previous studies were variable in terms of the most frequent treatments used by physiotherapists, showing various ranges of used treatments that were different between countries. In New Zealand, for example, Hendrick $e t a \hat{l}^{2}$ found that the most frequently prescribed physiotherapy treatments were spinal mobilisation (92.9\%), postural advice (91.8\%) and spinal stabilisation exercise $(84.7 \%)$. In Canada, Simmonds $e t a \vec{l}^{31}$ reported that included physiotherapists 
had received postprofessional training in manual therapy $(80 \%)$, the McKenzie Method (34\%) and chronic pain management (25\%). In Brazil, Magalhães $e t a l^{34}$ reported that $44 \%$ of physiotherapists used specific treatments such as global postural re-education (14\%), osteopathy $(10 \%)$ and motor control exercises (6\%). Meanwhile, in Thailand, Pensri et $a l^{35}$ reported that physiotherapists frequently used hot packs $(64 \%)$, ultrasound $(61 \%)$ and mechanical traction $(61 \%)$. The results of our study were different from those of the aforementioned studies, wherein physiotherapists in SA commonly used home exercises $(87.1 \%)$, patient education $(82 \%)$ and specific back exercises $(80.6 \%)$.

The differences between previous studies and our study in terms of the treatments most frequently used by physiotherapists could be explained by several factors such as the differences in demographic variables or professional backgrounds in the included sample. For example, the study conducted by Hendrick $e t a l^{22}$ included only manipulative and sports physiotherapists and did not consider other physiotherapy specialities. This was different from our study, as we included physiotherapists with any professional backgrounds or specialities. Another possible reason that may explain the differences between studies is the survey structure in terms of the questions related to the physiotherapy treatments and the number of questions that were asked. For example, Magalhães $e t$ $a \hat{l}^{34}$ and Simmonds $e t a l^{31}$ focused on only a small number of potential physiotherapy treatments for LBP, whereas in our study, we included a survey with 20 questions regarding the potential treatments that may be used in physiotherapy practice. In summary, the frequent treatments used by physiotherapists in SA are different from those in other countries. What is more, we found that there were large percentages of physiotherapists who used other treatments that were completely passive (eg, electrotherapy, soft tissue release, spinal mobilisation or manipulation) or related to traditional practices (eg, acupuncture or cupping therapy).

\section{The association between physiotherapists' pain attitudes and beliefs and treatment selection}

Several studies investigated the association between physiotherapists' pain attitudes and beliefs and the use of LBP guideline recommendations in terms of work, activity and bed rest, but no studies have investigated the association with treatment selection (such as exercises, manual therapy, electrotherapy, cognitive functional therapy and so on). Bishop $e t a l^{30}$ reported that physiotherapists in the UK with high biomedical and low biopsychosocial scores were less likely to follow guideline recommendations for LBP management (such as advice to remain off work) than were those with high biopsychosocial and low biomedical scores. Simmonds et $a l^{p 1}$ found that physiotherapists in Canada with stronger biomedical treatment orientation towards LBP and those who had received special training in manual therapy were likely to be more restricted in terms of encouraging patients with LBP to return to work and advising them to perform their normal activities than their counterparts who had stronger biopsychosocial treatment orientation and/or who had received special training in chronic pain management. Hendrick et $a l^{2}$ found that the practice of manipulative and sports physiotherapists in New Zealand who had lower biomedical treatment orientation towards LBP, those who had seen many LBP cases and those who had postprofessional qualifications were more likely (in line with guideline recommendations) to inform clinical decisions for managing individuals with LBP. According to a systematic review conducted by Gardner et al, ${ }^{13}$ the higher the biomedical orientation, the higher the belief that a return to work or normal life activities is a threat to patients with LBP, leading those therapists to avoid advising an early return to work and normal life activities.

In our study, physiotherapists who had higher scores in biomedical treatment orientation were more likely to use treatments such as specific back exercises, electrotherapy, soft tissue release, hydrotherapy, massage, lumbar supports and acupuncture. However, those who had higher scores in biopsychosocial treatment orientation were more likely to use treatments such as cognitive functional therapy and less likely to use lumbar supports. This suggests that the stronger the physiotherapists' biomedical treatment orientation towards NSCLBP, the higher the possibility that they would use biomedically passive treatments. Biomedical treatment orientation refers to the belief that pain and disability are caused only by specific structural diseases or dysfunctions, with the result that patients are classified based on the severity of tissue damage and treatments are selected to address only these dysfunctions. This may lead to paying less attention to psychological and social factors that could be involved, in addition to biomechanical factors as predisposing factors for the LBP condition. Physiotherapists, therefore, should be aware of their pain attitudes and beliefs, which may influence their choice of LBP management.

Another important aspect is the patients' perspective in terms of their pain attitudes and beliefs. Indeed, the involvement of patients with LBP in management has been found to be an essential component. ${ }^{36}$ According to the systematic review, there was strong evidence that the beliefs of healthcare professionals towards LBP were associated with the beliefs of their patients and moderate evidence that high levels of fear avoidance in healthcare professionals were associated with high levels of fear avoidance in their patients with LBP. ${ }^{14}$ Therefore, physiotherapists and all other healthcare professionals must be aware of the association between their pain attitudes and beliefs and the pain attitudes and beliefs of their patients with LBP.

This study found that physiotherapists who had received special training in LBP were more likely to have a higher score on the biopsychosocial subscale than were counterparts who had not received special training in LBP. Meanwhile, other studies also found changes in pain attitudes and beliefs among undergraduate physiotherapy students 
who had obtained a teaching module on chronic $\mathrm{LBP}^{37}$ or physiotherapists who had received specific training in cognitive functional therapy. ${ }^{38}$ Furthermore, these studies reported that their participants expressed confidence in their ability to manage individuals with LBP within biopsychosocial dimensions. Thus, these results show the importance of providing training on the biopsychosocial model for physiotherapists to improve their skills and confidence in understanding the complexity and management of LBP.

\section{Study strengths and limitations}

To the best of our knowledge, this is the first study to investigate physiotherapists' pain attitudes and beliefs towards NSCLBP and their association with treatment selection in SA. One strength of this study was that a sample size calculation was performed and the required number of participants was achieved. The reliability of the PABS-PT survey that was used to identify physiotherapists' pain attitudes and beliefs was acceptable. Meanwhile, another strength is the investigation into the association between pain attitudes and beliefs and the selection of various potential treatments (20 different treatments) that may be used by physiotherapists to manage NSCLBP.

There were some limitations to the current study. One of the limitations is the potential sampling bias due to the use of convenience sampling, which might not be representative of the entire physiotherapists working in SA. However, an attempt was made to reduce the sampling bias by distributing the survey to a large number of physiotherapists working in clinical and academic settings with the consideration of physiotherapists with any nationalities, as well as distributing the survey to all main geographical regions in SA. Another limitation is the fact that the completion rate of the survey in this study was relatively low (304/514; $59.14 \%$ ), wherein $40.86 \%$ of the received responses contained only demographic information; therefore, these responses were excluded from the analysis. Furthermore, the survey was distributed through social media platforms and WhatsApp, which makes it impossible to estimate the response rate. Meanwhile, another limitation is that the accuracy of the data is limited due to the use of a self-report survey, although it is considered to be a valid method of evaluating beliefs and attitudes. The final limitation is related to the statistical analysis where the large number of comparisons used in the current study may lead to some false-positive findings. Therefore, the current results should be interpreted with caution.

\section{CONCLUSIONS}

The biomedical and biopsychosocial treatment orientations of physiotherapists in SA were relatively low. The most frequent treatment strategies used by physiotherapists for NSCLBP were home exercises and patient education. However, some passive and traditional treatments which are not recommended by clinical practice guidelines for the management of LBP continue to be used frequently by physiotherapists in SA. This study has confirmed that physiotherapists' pain attitudes and beliefs are significantly associated with treatment selection when managing individuals with NSCLBP. Further research through which to better understand and facilitate the implementation of best evidence-based practice and clinical practice with the consideration of the biopsychosocial model in universities' curricula is a priority, particularly in countries in which the biopsychosocial model is rarely considered in the healthcare and education/university systems.

\section{Author affiliations}

${ }^{1}$ Physiotherapy Department, Faculty of Applied Medical Sciences, Umm Al-Qura University, Mecca, Saudi Arabia

${ }^{2}$ NHMRC Centre of Clinical Research Excellence in Spinal Pain, Injury and Health, School of Health and Rehabilitation Sciences, University of Queensland, Brisbane, Queensland, Australia

${ }^{3}$ Department of Physiotherapy, College of Applied Medical Sciences, Taif University, Taif, Saudi Arabia

${ }^{4}$ Department of Physical Therapy and Health Rehabilitation, College of Applied Medical Sciences, Prince Sattam bin Abdulaziz University, Al Kharj, Saudi Arabia ${ }^{5}$ Physiotherapy Department, Medical Rehabilitation Hospital, Medina, Saudi Arabia

Twitter Mansour Abdullah Alshehri @mansourpt and Hosam Alzahrani @Alzahrani_ Hosam

Acknowledgements The authors would like to thank the volunteers who helped to distribute the study survey to physiotherapists in Saudi Arabia. The authors also would like to thank all participants who took part in the study.

Contributors MAA conceived the study. MAA, HA, MA, AA and OK contributed in the survey design, data collection and data analysis. All authors contributed to the interpretation of data and intellectual revised multiple drafts. MAA and HA drafted the manuscript. All authors have approved the final version of the manuscript.

Funding The authors have not declared a specific grant for this research from any funding agency in the public, commercial or not-for-profit sectors.

Competing interests None declared.

Patient and public involvement Patients and/or the public were not involved in the design, or conduct, or reporting, or dissemination plans of this research.

Patient consent for publication Not required.

Ethics approval Ethical approval was obtained for the study from the Physiotherapy Research Committee, Faculty of Applied Medical Sciences, Umm Al-Qura University, Mecca, Saudi Arabia.

Provenance and peer review Not commissioned; externally peer reviewed.

Data availability statement The data sets used and analysed during the current study are available from the corresponding author on reasonable request.

Open access This is an open access article distributed in accordance with the Creative Commons Attribution Non Commercial (CC BY-NC 4.0) license, which permits others to distribute, remix, adapt, build upon this work non-commercially, and license their derivative works on different terms, provided the original work is properly cited, appropriate credit is given, any changes made indicated, and the use is non-commercial. See: http://creativecommons.org/licenses/by-nc/4.0/.

\section{ORCID iDs}

Mansour Abdullah Alshehri http://orcid.org/0000-0002-0294-9856

Hosam Alzahrani http://orcid.org/0000-0002-4383-115X

\section{REFERENCES}

1 Hoy D, Bain C, Williams G, et al. A systematic review of the global prevalence of low back pain. Arthritis Rheum 2012;64:2028-37.

2 Balagué F, Mannion AF, Pellisé F, et al. Non-specific low back pain. Lancet 2012;379:482-91.

3 Maetzel A, Li L. The economic burden of low back pain: a review of studies published between 1996 and 2001. Best Pract Res Clin Rheumatol 2002;16:23-30. 
4 Gore M, Sadosky A, Stacey BR, et al. The burden of chronic low back pain: clinical comorbidities, treatment patterns, and health care costs in usual care settings. Spine 2012;37:668-77.

5 Hoy D, Brooks P, Blyth F, et al. The epidemiology of low back pain. Best Pract Res Clin Rheumatol 2010;24:769-81.

6 Stefane T, dos Santos AM, Marinovic A, et al. Chronic low back pain: pain intensity, disability and quality of life. Acta Paul Enferm 2013;26:14-20.

7 Valat J-P. Factors involved in progression to chronicity of mechanical low back pain. Joint Bone Spine 2005;72:193-5.

8 Walker BF. The prevalence of low back pain: a systematic review of the literature from 1966 to 1998. J Spinal Disord 2000;13:205-17.

9 Maniadakis N, Gray A. The economic burden of back pain in the UK. Pain 2000;84:95-103.

10 Leung L. Pain catastrophizing: an updated review. Indian J Psychol Med 2012;34:204-17.

11 Waddell G, Newton M, Henderson I, et al. A Fear-Avoidance beliefs questionnaire $(\mathrm{FABQ}$ ) and the role of fear-avoidance beliefs in chronic low back pain and disability. Pain 1993;52:157-68.

12 Meucci RD, Fassa AG, Faria NMX. Prevalence of chronic low back pain: systematic review. Rev Saúde Pública 2015;49:1.

13 Gardner T, Refshauge K, Smith L, et al. Physiotherapists' beliefs and attitudes influence clinical practice in chronic low back pain: a systematic review of quantitative and qualitative studies. J Physiother 2017;63:132-43.

14 Darlow B, Fullen BM, Dean S, et al. The association between health care professional attitudes and beliefs and the attitudes and beliefs, clinical management, and outcomes of patients with low back pain: a systematic review. Eur J Pain 2012;16:3-17.

15 Widerström B, Rasmussen-Barr E, Boström C. Aspects influencing clinical reasoning and decision-making when matching treatment to patients with low back pain in primary healthcare. Musculoskelet Sci Pract 2019;41:6-14.

16 Ostelo RWJG, Stomp-van den Berg SGM, Vlaeyen JWS, et al. Health care provider's attitudes and beliefs towards chronic low back pain: the development of a questionnaire. Man Ther 2003;8:214-22.

17 Koes BW, van Tulder M, Lin C-WC, et al. An updated overview of clinical guidelines for the management of non-specific low back pain in primary care. Eur Spine J 2010;19:2075-94.

18 National Institute for Health and Care Excellence. Low back pain and sciatica in over 16S: assessment and management. NICE, guideline [NG59]. London: National Institute for Health and Care Excellence, 2016.

19 Alghadir A, Anwer S. Prevalence of musculoskeletal pain in construction workers in Saudi Arabia. ScientificWorldJournal 2015;2015:ID529873.

20 Alshami AM. Prevalence of spinal disorders and their relationships with age and gender. Saudi Med J 2015;36:725-30.

21 Attar SM. Frequency and risk factors of musculoskeletal pain in nurses at a tertiary centre in Jeddah, Saudi Arabia: a cross sectional study. BMC Res Notes 2014;7:61.

22 Abdulmonem A, Hanan A, Elaf A, et al. The prevalence of musculoskeletal pain \& its associated factors among female Saudi school teachers. Pak J Med Sci 2014;30:1191.
23 Muaidi QI, Shanb AA. Prevalence causes and impact of work related musculoskeletal disorders among physical therapists. J Back Musculoskelet Rehabil 2016;29:763-9.

24 AlBedah A, Khalil M, Elolemy A, et al. The use of wet cupping for persistent nonspecific low back pain: randomized controlled clinical trial. J Altern Complement Med 2015;21:504-8.

25 Alshehri MA, Alalawi A, Alhasan $\mathrm{H}$, et al. Physiotherapists' behaviour, attitudes, awareness, knowledge and barriers in relation to evidencebased practice implementation in Saudi Arabia: a cross-sectional study. Int J Evid Based Healthc 2017;15:127-41.

26 Awwad WM, Alfayez SM, Bin Dous AN, et al. Knowledge around back pain and spinal disorders among Saudi patients: a crosssectional study. J Pak Med Assoc 2017;67:1228-31.

27 Houben RMA, Ostelo RWJG, Vlaeyen JWS, et al. Health care providers' orientations towards common low back pain predict perceived harmfulness of physical activities and recommendations regarding return to normal activity. Eur J Pain 2005;9:173-83.

28 Bishop A. Pain attitudes and beliefs scale (PABS). J Physiother 2010;56:279.

29 Saudi Commission for Health Specialties. Health workforce, 2018. Available: https://www.scfhs.org.sa/Media/DigitalLibrary/ DocumentLibrary/OtherPublications/Pages/default.aspx [Accessed 3 Aug 2019].

30 Bishop A, Foster NE, Thomas E, et al. How does the self-reported clinical management of patients with low back pain relate to the attitudes and beliefs of health care practitioners? A survey of UK general practitioners and physiotherapists. Pain 2008;135:187-95.

31 Simmonds MJ, Derghazarian T, Vlaeyen JWS. Physiotherapists knowledge, attitudes, and intolerance of uncertainty influence decision making in low back pain. Clin J Pain 2012;28:467-74.

32 Hendrick P, Mani R, Bishop A, et al. Therapist knowledge, adherence and use of low back pain guidelines to inform clinical decisions--a national survey of manipulative and sports physiotherapists in New Zealand. Man Ther 2013:18:136-42.

33 Innes SI, Werth PD, Tuchin PJ, et al. Attitudes and beliefs of Australian chiropractors' about managing back pain: a crosssectional study. Chiropr Man Therap 2015;23:17.

34 Magalhães MO, Costa LOP, Cabral CMN, et al. Attitudes and beliefs of Brazilian physical therapists about chronic low back pain: a crosssectional study. Rev Bras Fisioter 2012;16:248-53.

35 Pensri P, Foster NE, Srisuk S, et al. Physiotherapy management of low back pain in Thailand: a study of practice. Physiother Res Int 2005;10:201-12

36 May S. Patients' attitudes and beliefs about back pain and its management after physiotherapy for low back pain. Physiother Res Int 2007;12:126-35.

37 Latimer J, Maher C, Refshauge K. The attitudes and beliefs of physiotherapy students to chronic back pain. Clin J Pain 2004;20:45-50.

38 Synnott A, O'Keeffe M, Bunzli S, et al. Physiotherapists report improved understanding of and attitude toward the cognitive, psychological and social dimensions of chronic low back pain after cognitive functional therapy training: a qualitative study. J Physiother 2016;62:215-21. 